\title{
Review: interventions focusing on patient behaviours in provider patient interactions improve diabetes outcomes
}

van Dam HA, van der Horst F, van den Borne B, et al. Provider-patient interaction in diabetes care: effects on patient self-care and outcomes. A systematic review. Patient Educ Couns 2003;51:17-28.

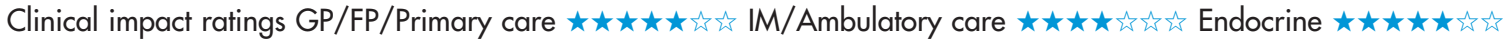

In patients with diabetes, do experimental modifications of provider patient interactions improve patient behaviours and health outcomes?

\section{METHODS}

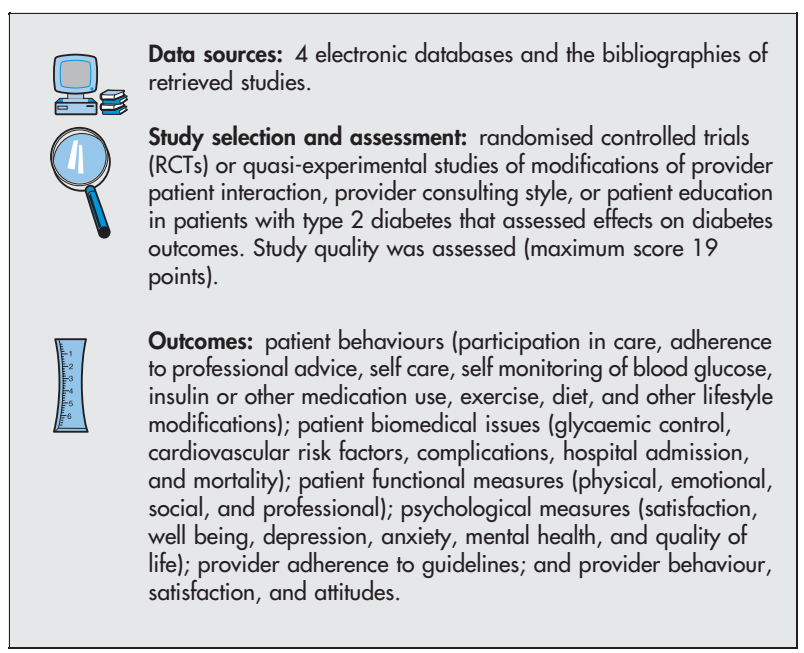

\section{MAIN RESULTS}

8 RCTs ( 4 on provider behaviour, 4 on patient behaviour) met the selection criteria. The quality score ranged from 13 to 19 points (mean 17.3 points). While 3 of 4 trials focusing on providers showed modification of clinicians' behaviour, trials focusing on patients showed more positive effects on such biomedical outcomes as glucose control, as well as self care and psychosocial outcomes (table).

\section{CONCLUSIONS}

In patients with diabetes, interventions targeting patient behaviour seem to have better results than those targeting clinician behaviour.

\section{Abstract and commentary also appear in ACP Journal Club.}

\begin{abstract}
Commentary
The systematic review by van Dam et al, and another one published later, ${ }^{1}$ support at least 2 conclusions: Firstly, providers with more patient centred behaviours (the result of multimodality interventions to change provider behaviour) enhance patient satisfaction with care. Patients appreciate clinicians who take their views and beliefs into account. Secondly, interventions empowering patients to participate actively in the encounter and to make informed choices on their own improve self efficacy measures and metabolic control (eg, haemoglobin $A_{1 c}$ levels). Patients do better when they get involved in the day to day management of their condition.

Most of the decisions that affect patient important outcomes in diabetes occur in the "patient space" (eg, diet and exercise choices, medication adherence, and self monitoring). Thus, the clinical encounter may be most effective when clinicians (and other members of the care team) take into account patient treatment goals, offer patients tools and support to problem solve in that "space," and provide extended and tailored support after the encounter.

Healthcare professionals in some practices have made efforts to redesign their delivery of diabetes care to include effective encounter strategies. ${ }^{2}$ Unfortunately, economic incentives exist to make encounters brief, which may hinder a participatory encounter style. ${ }^{3}$ It may follow that brief encounters with limited patient participation lead to poor patient outcomes, including preventable diabetes complications and their associated costs in suffering and healthcare dollars. Other barriers include discontinuity of care, lack of provider motivation, and lack of skills to support patient participation.

While identifying large knowledge gaps in need of research, this review offers hope that implementing multimodality interventions targeting patients and their healthcare providers may lead to more effective and satisfactory diabetes encounters.

Victor M Montori, MD, MSc Mayo Clinic College of Medicine Rochester, Minnesota, USA

1 Michie S, Miles J, Weinman J. Patient Educ Couns 2003;51:197-206.

2 Bodenheimer T. Dis Manag 2003;6:63-71.

3 Kaplan SH, Gandek B, Greenfield S, et al. Med Care 1995;33:1176-87.
\end{abstract}

For correspondence: Dr H A van Dam, Maastricht University, Maastricht, The Netherlands. rnhprk09@hag.unimaas.nl

Source of funding: not stated.

Interventions on provider patient interactions in type 2 diabetes at 6 months to 6 years

Number of trials showing improvement, no improvement, or uncertain improvement

Outcomes

Biomedical (see outcomes section)

Self care (see outcomes section)

Psychosocial (see outcomes section)
Focus of intervention

Provider behaviour

Patient behaviour

Provider behaviour

Patient behaviour

Provider behaviour

Patient behaviour

\begin{tabular}{lll}
\hline Improvement & No improvement & Uncertain improvement \\
1 & 3 & 0 \\
4 & 0 & 0 \\
0 & 3 & 1 \\
2 & 2 & 0 \\
0 & 1 & 3 \\
3 & 0 & 1
\end{tabular}

